\title{
Antibodies to post-translationally modified insulin in type 1 diabetes
}

\author{
Rocky Strollo $^{1,2}$ - Chiara Vinci ${ }^{1,2}$ • Mayda H. Arshad ${ }^{1}$ • David Perrett ${ }^{3}$. \\ Claudio Tiberti ${ }^{4}$ - Francesco Chiarelli ${ }^{5}$. Nicola Napoli ${ }^{2,6}$ • Paolo Pozzilli ${ }^{2,7}$. \\ Ahuva Nissim ${ }^{1}$
}

Received: 17 May 2015 / Accepted: 11 August 2015 / Published online: 8 September 2015

(C) Springer-Verlag Berlin Heidelberg 2015

\begin{abstract}
Aim/hypothesis Insulin is the most specific beta cell antigen and a potential primary autoantigen in type $1 \mathrm{di}$ abetes. Insulin autoantibodies (IAAs) are the earliest marker of beta cell autoimmunity; however, only slightly more than $50 \%$ of children and even fewer adults newly diagnosed with type 1 diabetes are IAA positive. The aim of this investigation was to determine if oxidative post-translational modification (oxPTM) of insulin by reactive oxidants associated with islet inflammation generates neoepitopes that stimulate an immune response in individuals with type 1 diabetes.

Methods oxPTM of insulin was generated using ribose and various reactive oxygen species. Modifications were analysed by SDS-PAGE, three-dimensional fluorescence and MS. Autoreactivity to oxPTM insulin (oxPTM-INS) was observed by ELISA and western blotting, using sera from participants
\end{abstract}

Paolo Pozzilli and Ahuva Nissim contributed equally to this study.

Electronic supplementary material The online version of this article (doi:10.1007/s00125-015-3746-x) contains peer-reviewed but unedited supplementary material, which is available to authorised users.

Paolo Pozzilli

p.pozzilli@unicampus.it

Ahuva Nissim

a.nissim@qmul.ac.uk

1 Centre for Biochemical Pharmacology, William Harvey Research Institute, Barts and The London School of Medicine and Dentistry, Queen Mary University of London, Charterhouse Square, London EC1M 6BQ, UK

2 Endocrinology \& Diabetes, University Campus Bio-Medico, via Alvaro del Portillo 21, 00128 Rome, Italy with type 1 or type 2 diabetes and healthy controls as probes. IAA was measured using the gold-standard radiobinding assay (RBA).

Results MS of oxPTM-INS identified chlorination of Tyr16 and Tyr26; oxidation of His5, Cys7 and Phe24; and glycation of Lys 29 and Phe 1 in chain B. Significantly higher binding to oxPTM-INS vs native insulin was observed in participants with type 1 diabetes, with $84 \%$ sensitivity compared with $61 \%$ sensitivity for RBA. oxPTM-INS autoantibodies and IAA co-existed in $50 \%$ of those with type 1 diabetes. Importantly $34 \%$ of those with diabetes who were IAA negative were oxPTMINS positive. Altogether, $95 \%$ of participants with type 1 diabetes presented with autoimmunity to insulin by RBA, oxPTM-INS or both. Binding to oxPTM-INS was directed towards oxPTM-INS fragments with slower mobility than native insulin.
3 BioAnalysis, William Harvey Research Institute, Barts and The London School of Medicine and Dentistry, Queen Mary University of London, London, UK

4 Department of Experimental Medicine, Sapienza University of Rome, Rome, Italy

5 Department of Pediatrics, University of Chieti, Ospedale Policlinico, Chieti, Italy

6 Division of Bone and Mineral Diseases, Washington University in St Louis, St Louis, MO, USA

7 Centre for Immunobiology, Blizard Institute, Barts and The London School of Medicine and Dentistry, Queen Mary University of London, London, UK 
Conclusion/interpretation These data suggest that oxPTMINS is a potential autoantigen in individuals with new-onset type 1 diabetes.

Keywords Insulin autoantibodies · Oxidative stress . Post-translational modification · Reactive oxygen species . Type 1 diabetes

$\begin{array}{ll}\text { Abbreviations } \\ \mathrm{Em}_{\max } & \text { Maximum emission } \\ \mathrm{Ex}_{\max } & \text { Maximum excitation } \\ \mathrm{HEL} & \text { Hen egg lysozyme } \\ \mathrm{HOCl} & \text { Hypochlorous acid } \\ \text { HOCl-INS } & \text { Insulin modified by hypochlorous acid } \\ \text { IAA } & \text { Insulin autoantibody } \\ \text { IMDIAB } & \text { Immunotherapy Diabetes } \\ \text { •OH } & \text { Hydroxyl radical } \\ \text { 'OH-INS } & \text { Insulin modified by the hydroxyl radical } \\ \text { oxPTM } & \text { Oxidative post-translational modification } \\ \text { oxPTM- } & \text { Oxidative post-translationally modified insulin } \\ \text { INS } & \\ \text { PTM } & \text { Post-translational modification } \\ \text { RBA } & \text { Radiobinding assay } \\ \text { ROS } & \text { Reactive oxygen species }\end{array}$

\section{Introduction}

Oxidative stress might be a critical player in the pathogenesis of type 1 diabetes. Hyperglycaemia and a large influx of metabolically active immune cells infiltrating the inflamed islets might result in the formation of high levels of reactive oxygen species (ROS) $[1,2]$. The key ROS that are known to be produced are the superoxide radical, hydrogen peroxide, the hydroxyl radical ( $\left.{ }^{\circ} \mathrm{OH}\right)$, hypochlorous acid $(\mathrm{HOCl})$, nitric oxide, peroxynitrite and oxidants derived from glycation as a result of hyperglycaemia. High levels of ROS may lead to oxidative post-translational modification (oxPTM) of beta cell self-proteins and the formation of neoepitopes. Neoepitopes are epitopes that have not been previously presented to the immune system $[3,4]$ and therefore escape immune tolerance and generate autoimmunity. Other intracellular events, such as increases in ROS and endoplasmic reticulum stress, impair beta cell autophagic activity [5]. This leads to apoptosis, inflammation, altered processing of beta cell antigens and accumulation of extracellular matrix, which are in turn both targets for ROS and further oxPTM.

Although this mechanism is well established in other autoimmune diseases such as coeliac disease and rheumatoid arthritis [6,7], there is scant evidence available for type 1 diabetes [8-12]. We have previously shown that patients with type 1 diabetes had increased levels of autoantibodies against collagen type II modified by ROS, and that this response was under the genetic control of the $H L A-D R B 1^{*} 04$ shared epitope alleles [13]. Although the effect of ROS in inducing autoimmunity towards beta-cell-specific antigens remains largely unknown, this role for ROS is supported by the evidence that antibodies against oxidised GAD are present in diabetes [11]. Insulin is the most specific antigen for pancreatic beta cells and a potential primary autoantigen in type 1 diabetes. Insulin autoantibodies (IAAs) have been reported since 1983 [14] and represent the earliest marker of beta cell autoimmunity [15]. However, only slightly more than half of children and even fewer adults with recent-onset type 1 diabetes are IAA positive $[16,17]$.

It is an enigma why tolerance to insulin malfunctions in type 1 diabetes. Our hypothesis is that oxPTM by oxidants associated with islet inflammation has a role in breaching tolerance to insulin in type 1 diabetes. To address this hypothesis, we have tested reactivity to native and oxPTM insulin (oxPTM-INS) in individuals with newly diagnosed type 1 diabetes, and compared the results with those from control participants using both cross-sectional and longitudinal designs.

\section{Methods}

Participants Serum samples were obtained from participating centres of the Immunotherapy Diabetes (IMDIAB) group. A population of young individuals diagnosed with type 1 diabetes according to ADA criteria was studied. Serum samples from participants with type 1 diabetes $(n=116)$ were collected at diagnosis, before the start of insulin therapy. For 69 patients, a second serum sample was obtained at the following time points after the start of insulin therapy: 3 months (19 samples); 3-6 months (16 samples); 6-9 months (19 samples); and 12 months (15 samples). Sera from 64 individuals with type 2 diabetes and 113 healthy individuals (45 schoolchildren and 68 adults) were used as controls. Features of the studied cohorts are reported in Table 1. This project was approved by the ethical committee at the University Campus Bio-Medico within the framework of the IMDIAB investigators' type 1 diabetes study, with informed consent signed by participants or their parents.

In vitro chemical modifications Human recombinant insulin $(1 \mathrm{mg} / \mathrm{ml})$ in PBS was incubated overnight at $37^{\circ} \mathrm{C}$ with the following agents: (1) $2 \mathrm{~mol} / \mathrm{l}$ ribose (Sigma, Gillingham, UK); (2) $9 \mathrm{mmol} / \mathrm{l} \mathrm{HOCl}$ (BDH, Oxford, UK); (3) $4.5 \mathrm{mmol} / \mathrm{l}$ $\mathrm{CuCl}_{2}$ (Sigma) and $9 \mathrm{mmol} / \mathrm{l}$ hydrogen peroxide (Sigma), which was used to produce ${ }^{\circ} \mathrm{OH}$ by the Fenton reaction. Hen egg lysozyme (HEL; Sigma) was also modified as above and used as the control antigen. Modification of insulin was monitored by $20 \%$ reducing SDS-PAGE and $20 \%$ native-PAGE, followed by staining with Coomassie blue (Sigma). 
Table 1 Clinical and biochemical features of the studied populations

\begin{tabular}{|c|c|c|c|c|c|}
\hline \multirow[t]{2}{*}{ Characteristic } & \multicolumn{2}{|l|}{ Type 1 diabetes } & \multirow[t]{2}{*}{ Type 2 diabetes $(n=64)$} & \multicolumn{2}{|l|}{ No diabetes } \\
\hline & At diagnosis $(n=116)$ & After insulin $^{\mathrm{a}}(n=69)$ & & Children $(n=45)$ & Adults $(n=68)$ \\
\hline Age, years & $13.9 \pm 0.8$ & $14.8 \pm 1.0$ & $59.5 \pm 3.4$ & $14.2 \pm 0.6$ & $38.0 \pm 1.4$ \\
\hline Ratio of male:female & 0.85 & 0.97 & 1 & 0.33 & 0.66 \\
\hline Blood glucose, mmol/1 & $21.4 \pm 0.7$ & $8.4 \pm 0.7$ & $8.6 \pm 0.4$ & $4.2 \pm 0.1$ & \\
\hline $\mathrm{HbA}_{1 \mathrm{c}}, \%(\mathrm{mmol} / \mathrm{mol})$ & $11.0 \pm 0.3(96 \pm 2.9)$ & $6.8 \pm 0.3(51 \pm 3.1)$ & $7.4 \pm 0.3(57 \pm 2.9)$ & $5.4 \pm 0.1(39 \pm 4.02)$ & \\
\hline BMI, $\mathrm{kg} / \mathrm{m}^{2}$ & $18.7 \pm 0.4$ & $18.7 \pm 0.5$ & $31.8 \pm 2.1$ & $21.4 \pm 0.8$ & \\
\hline
\end{tabular}

Data are means \pm SE, unless otherwise stated

${ }^{\text {a }}$ See "Methods" for disease duration

Three-dimensional (3D) fluorescence 3D scanning fluorescence spectra were obtained using a Hitachi F-4500 spectrofluorometer (Tokyo, Japan). Samples were briefly centrifuged prior to scanning to remove aggregated material. Simultaneous excitation (200-800 $\mathrm{nm}$ ) and emission (200-800 nm) spectra were recorded.

MS Samples were desalted with StageTip C18 and analysed by MALDI-TOF in positive, linear mode, mass range $\mathrm{m} / \mathrm{z}$ from 2,000 to 25,000. An aliquot of the samples was reduced with DTT, digested with Glu-C (Calbiochem, Merck, Darmstadt, Germany), desalted using StageTip C18 and submitted to MALDI-TOF (MS) and MALDI-TOF-TOF (MS/MS) analysis and nano-electrospray ionisation tandem MS.

Detection of autoantibodies to oxPTM-INS An ELISA was performed using native insulin and oxPTM-INS or control native HEL and oxPTM-HEL as targets. Briefly, ELISA plates (Nunc, London, UK) were coated with $10 \mu \mathrm{g} / \mathrm{ml}$ modified or native protein in $0.05 \mathrm{M}$ carbonate/bicarbonate buffer $(\mathrm{pH} 9.6)$ at $4{ }^{\circ} \mathrm{C}$ overnight. Plates were then washed three times with PBS. After blocking for $2 \mathrm{~h}$ with 5\% BSA in $0.5 \%$ Tween-PBS, $100 \mu$ of 1:200-diluted serum samples in $5 \%$ BSA in $0.5 \%$ Tween-PBS were added to each well, followed by $2 \mathrm{~h}$ incubation at room temperature. Plates were then washed with PBS plus $0.1 \%$ Tween, followed by three washes with PBS. Anti-human IgG-HRP conjugated antibodies (Sigma) were then added at 1:1,000 dilution in 5\% BSA in $0.5 \%$ Tween-PBS for another $2 \mathrm{~h}$ incubation. The ELISA

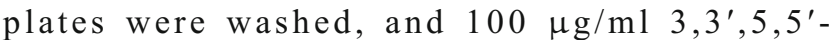
tetramethylbenzidine substrate (Sigma) in $100 \mathrm{mmol} / 1$ sodium acetate ( $\mathrm{pH}$ 6.0) was added. Subsequently, the reaction was stopped with $1 \mathrm{~mol} / \mathrm{l}$ sulphuric acid. Absorbance was measured at $450 \mathrm{~nm}$ using a GENios plate reader and Magellan software (Tecan, Reading, UK).

The ELISA absorbance values obtained for HEL and oxPTM-HEL were used as background controls that were subtracted from the absorbance values of native insulin and oxPTM-INS, respectively. In addition, to control assay fluctuation, binding to insulin, oxPTM-INS, HEL and oxPTM-HEL was tested for each individual sample on the same plate. Each assay included known positive or negative reference control samples. Longitudinal samples obtained from the same individuals (before and after insulin treatment) were tested on the same plate. Levels of oxPTM-INS antibodies above the 99th percentile of the healthy individuals were defined as the ELISA cut-off.

A competitive ELISA was performed to assess the binding specificity of serum to oxPTM-INS. The competitive ELISA was carried out in a similar manner as above, except that the serum samples were pre-incubated for $2 \mathrm{~h}$ or overnight, with and without $10 \mu \mathrm{g} / \mathrm{ml}$ native insulin or oxPTM-INS as the competitor, before adding the serum samples to the coated ELISA plate.

Radiobinding assay (RBA) for detection of IAA IAAs were measured by RBA using a modified radioimmunoprecipitation assay initially described by Williams and coworkers [18]. Briefly, $20 \mu \mathrm{l}$ serum was incubated for 2 days at $4^{\circ} \mathrm{C}$ in the presence of human 125-I insulin. Immune complexes were then precipitated by using $50 \%$ protein $\mathrm{A} / \mathrm{G}-\mathrm{Sepharose}$. After several washings, bound 125-I insulin was measured in a beta counter. Results were expressed as an index defined as follows: (sample cpm - negative standard control cpm) / (positive standard control cpm - negative standard control cpm). Serum samples with an index $>0.002$ were considered to be positive. The assay limit of positivity was calculated according to $>99$ th percentile values of 150 healthy control sera. This assay achieved $46 \%$ sensitivity and $100 \%$ specificity at the Islet Autoantibody Standardization Proficiency Workshop in 2012.

Western blot PAGE was run as described above and proteins were transferred to nitrocellulose using standard protocols. Membranes were blocked in 5\% dry milk powder (Marvel) for $2 \mathrm{~h}$, followed by overnight incubation at $4{ }^{\circ} \mathrm{C}$ with serum samples diluted $1: 1,000$ in 5\% dry milk powder. After washing twice for $5 \mathrm{~min}$ in $0.5 \%$ Tween-PBS and for $5 \mathrm{~min}$ in PBS, membranes were incubated for $1 \mathrm{~h}$ with anti-IgG HRP 
(Sigma), diluted 1:1,000 in 5\% dry milk powder. Membranes were then washed twice for $10 \mathrm{~min}$ in $0.5 \%$ Tween-PBS and for $10 \mathrm{~min}$ in PBS before incubating with $1 \mathrm{ml}$ Luminata Forte Western HRP (Sigma). Bands were detected using the FluorChem E System (ProteinSimple, Santa Clara, CA, USA).

Statistical analysis Statistical analyses were performed using Prism (GraphPad, San Diego, CA, USA) and SPSS (SPSS, Chicago, IL, USA). Differences in antibody levels between the groups were tested using the Mann-Whitney $U$ (Wilcoxon) test, and data are presented as medians \pm SE. Longitudinal changes in antibody binding were evaluated using the Wilcoxon paired test. Pearson's correlation test was used to assess the association between reactivity to oxPTM-INS and metabolic control. To determine diagnostic discrimination between participants with type 1 diabetes, type 2 diabetes and healthy controls, we used the 99th percentile of the healthy individuals as the cut-off point for absorbance units to construct a contingency table of positive autoantibodies against the clinical diagnosis (type 1 diabetes vs healthy control; type 1 diabetes vs type 2 diabetes) and tested it using Fisher's exact test. The ELISA intra-assay CV was calculated as the SD of a set of triplicate measurements divided by the mean of the serum samples. The inter-assay CV was calculated similarly for samples assessed in different experiments performed by different operators.

\section{Results}

PAGE analysis of native and oxPTM-INS Using SDSPAGE analysis, ROS-modified insulin (oxPTM-INS) and native insulin migrated to the region of $5 \mathrm{kDa}$ with no significant differences in mobility, although a smear above the major $5 \mathrm{kDa}$ band was observed for oxPTM-INS (Fig. 1a, SDSPAGE). In non-denaturing native-PAGE, differences emerged between native and modified insulin. After glycation by ribose, a clear shift in the position of the insulin band appeared, together with two additional bands, all with slower mobility. Exposure of insulin to the ${ }^{\circ} \mathrm{OH}$-generating system and $\mathrm{HOCl}$ induced the appearance of additional and slower mobility bands, in conjunction with a smear of protein through the entire line, suggesting fragmentation (Fig. 1b).

3D-fluorescence profile of native and oxPTM-INS To determine the effect of ROS modifications on the structural changes of insulin, we performed a 3D-fluorescence profile study of both native and oxPTM-INS (Fig. 1c-f). Intrinsic fluorescence was detectable in native insulin, with a maximum excitation $\left(\mathrm{Ex}_{\max }\right)$ of $279 \mathrm{~nm}$ and maximum emission $\left(\mathrm{Em}_{\max }\right)$ of $318 \mathrm{~nm}$, possibly attributed to tyrosine residues. $\mathrm{Ex}_{\max }$ and $\mathrm{Em}_{\max }$ shifted to 326 and $452 \mathrm{~nm}$, respectively, after modification of insulin with ribose, and to 336 and $431 \mathrm{~nm}$, respectively, after modification with $\mathrm{HOCl}$, suggesting changes in insulin structure. Modification with ${ }^{\circ} \mathrm{OH}$ resulted in the loss of fluorescence and an increase in light scattering, suggesting aggregation of the native molecules.

MS analysis of native and oxPTM-INS Native and modified insulin were analysed by high-resolution MS. Native insulin showed a peak at m/z 5,808 Da, corresponding to the molecular mass of insulin. After glycation by ribose, four additional peaks appeared with mass differences of $132 \mathrm{Da}$, indicating the addition of ribose molecules to insulin and corresponding to the mono-, di-, tri- and tetra-glycated insulin forms. Exposure to $\mathrm{HOCl}$ induced the development of additional peaks at lower $\mathrm{m} / \mathrm{z}$ with respect to the native form, indicating the formation of degradation products induced by the reaction with the oxidant. A peak at $\mathrm{m} / \mathrm{z} 5,824 \mathrm{Da}(+16)$ was shown for insulin modified by ${ }^{\circ} \mathrm{OH}$ ( $\left.\mathrm{OH}-\mathrm{INS}\right)$; the mass difference between native insulin and ${ }^{\circ} \mathrm{OH}-\mathrm{INS}$ was $16 \mathrm{Da}$, which corresponds to one oxidation site (Fig. 2a-d). MS/MS analysis suggested that the main oxidative changes involved Phe1 and Lys29 (glycation); His5, Cys7 and Phe24 ( ${ }^{\circ} \mathrm{OH}$ and HOCl); and Tyr16 and Try26 (HOCl) in chain B (Fig. 2e, Table 2).

\section{Antibody binding to oxPTM-INS in patients with type 1} diabetes and control individuals Native insulin and oxPTMINS were used as targets in ELISA to assess antibody binding to native insulin vs oxPTM-INS in sera from individuals with type 1 diabetes. As controls, we used samples from a group of healthy schoolchildren and a group of adults. To avoid interference with antibodies against exogenous insulin, samples from patients with type 1 diabetes (mean \pm SE age 13.90土 0.77 years) were taken at diagnosis, before the patients started insulin therapy (Table 1). Binding to insulin modified by $\mathrm{HOCl}$ (HOCl-INS) and ${ }^{\circ} \mathrm{OH}-\mathrm{INS}$ was significantly higher than binding to native insulin. Reactivity to native insulin and oxPTM-INS was detected in $30 \%$ and $84 \%$, respectively, of patients with type 1 diabetes, with median absorbances of $0.043 \pm 0.012,0.183 \pm 0.012$ and $0.231 \pm 0.013$ for binding to native insulin, $\mathrm{HOCl}-\mathrm{INS}$ and ${ }^{\circ} \mathrm{OH}-\mathrm{INS}$, respectively $(p<0.0001$, Fig. 3a). Binding to glycated insulin was significantly lower compared with native insulin (absorbance 0.007 $\pm 0.006, p<0.0001$, data not shown). Antibodies to the control antigens HEL and oxPTM-HEL were present in only $5 \%$ and $3.5 \%$ of participants with type 1 diabetes, respectively (Electronic supplementary material [ESM] Table 1). Binding of HOCl-INS and ${ }^{\circ} \mathrm{OH}-\mathrm{INS}$ was higher in sera from participants with type 1 diabetes than in that from participants with type 2 diabetes and healthy individuals $(p<0.0001)$. The sensitivity and specificity of oxPTM-INS reactivity in patients with type 1 diabetes were $84 \%$ and $99 \%$, respectively, 


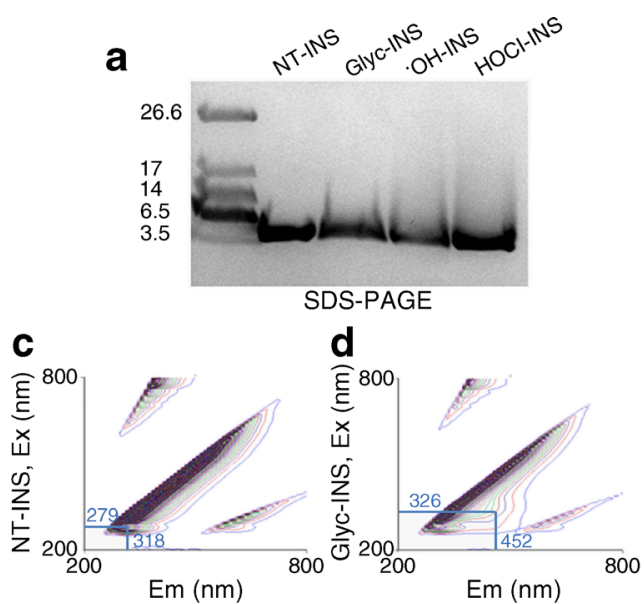

Fig. 1 Analysis of native insulin (NT-INS) and oxPTM-INS. (a) SDSPAGE and (b) native-PAGE analysis of oxPTM-INS: glycation by ribose (Glyc-INS), modification by ${ }^{\circ} \mathrm{OH}$ ('OH-INS) and $\mathrm{HOCl}$ (HOCl-INS). There were no clear changes in molecular mass between NT-INS and oxPTM-INS. The position of the molecular mass markers (in $\mathrm{kDa}$ ) is shown in (a). Native-PAGE demonstrated a clear reduction in the mobility of insulin after glycation by ribose, as shown by two bands with slower mobility. Exposure of insulin to the $\mathrm{HOCl}-$ and ${ }^{\circ} \mathrm{OH}$-generating systems

compared with healthy schoolchildren, and $66 \%$ and $99 \%$, respectively, compared with patients with type 2 diabetes.

To exclude the possibility that serum reactivity was due to metabolic alteration at the time of the clinical onset of diabetes, binding in 69 patients with type 1 diabetes was evaluated longitudinally after insulin treatment (Fig. 3c-e). Insulin therapy induced a slight increase in binding to native insulin (median absorbance $0.040 \pm 0.014$ vs $0.083 \pm 0.026 ; p<0.0001$ ) and HOCl-INS (median absorbance $0.187 \pm 0.019$ vs $0.262 \pm$ 0.024; $p=0.007$ ), but not to ${ }^{\circ} \mathrm{OH}-\mathrm{INS}$ (data not shown). Overall, binding to oxPTM-INS remained significantly higher than to native insulin $(p<0.0001)$. Intra-assay CV of triplicates was $<8 \%$ (mean $4 \%, n=10$ ). Inter-assay CVs were $<10 \%$ (mean 6\%) and $<13 \%$ (mean 6\%) for native insulin and oxPTM-INS $(n=12)$, respectively.

\section{Comparison between oxPTM-INS ELISA and RBA At} diagnosis, reactivity to oxPTM-INS ("OH-INS and/or HOClINS) and IAA in RBA was detected in $84 \%$ and $61 \%$ of participants with type 1 diabetes, respectively (Fig. 3a, b). The sensitivity of oxPTM-INS antibodies was $84 \%$ vs $61 \%$ for IAA measured by RBA $(p=0.0001)$, while specificity was 99\% for both assays. oxPTM-INS antibodies by ELISA and IAA by RBA coexisted in $50 \%$ of individuals with type 1 diabetes, but oxPTM-INS antibodies were able to detect an additional $34 \%$ of participants who were IAA negative. Overall, $82.7 \%$ of participants who were positive on RBA were also oxPTM-INS positive on ELISA, while $17.3 \%$ who were positive on RBA were oxPTM negative on ELISA. The combined measurement of IAA by RBA and oxPTM-INS
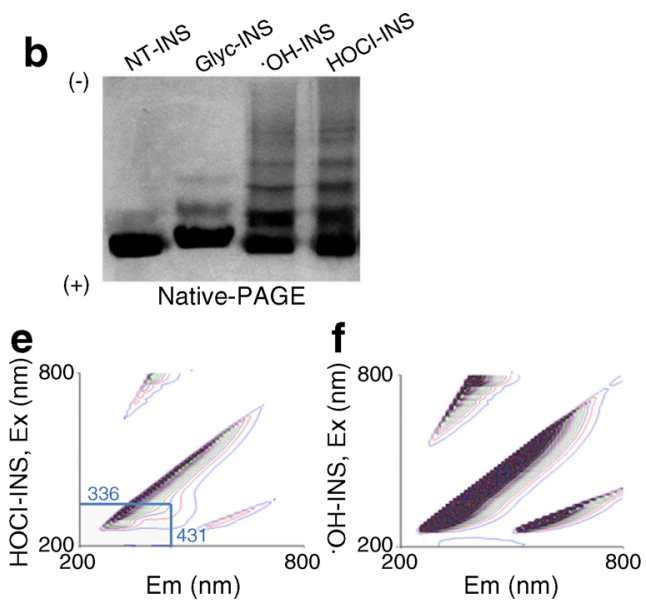

induced the appearance of additional and slower mobility bands, and a smear of protein through the entire line suggesting fragmentation. $(\mathbf{c}-\mathbf{f})$ 3D fluorescence profile of NT-INS and oxPTM-INS. Modification of insulin with ribose (d) or $\mathrm{HOCl}$ (e) resulted in a substantial shift in the $\mathrm{Em}_{\max }$ and $\mathrm{Ex}_{\max }$ wavelengths (indicated by blue lines and numbers) compared with NT-INS. (f) Modification with ${ }^{\circ} \mathrm{OH}$ resulted in loss of the native fluorescence and increased light scattering, suggesting aggregation of the native molecules

antibody ELISA raised the detection of insulin autoimmunity to $95 \%$ of participants with new-onset type 1 diabetes.

Antibody binding specificity to oxPTM-INS by competitive ELISA and western blot A competitive displacement assay was performed to evaluate serum binding specificities to oxPTM-INS by pre-incubating sera with either native insulin or oxPTM-INS (Fig. 4). When type 1 diabetes sera were preincubated with an excess amount of native insulin, no significant displacement occurred and the competitive assay showed only a $25 \%$ lower absorbance compared with the direct binding oxPTM-INS assay. In contrast, pre-incubation of sera with an excess of oxPTM-INS ("OH-INS or HOCl-INS) led to a strong reduction in binding ( $88 \%$ mean reduction in absorbance), indicating that antibody specificities are mainly to oxPTM-INS. Western blot analysis was then performed on a range of serum samples that exhibited the strongest binding in ELISAs. Weak binding to a fragment corresponding to native insulin was evident, but this was lost after glycation. Binding to ${ }^{\circ} \mathrm{OH}-\mathrm{INS}$ and $\mathrm{HOCl}-\mathrm{INS}$ was stronger and directed towards a diffused fragment that had slower mobility than native insulin. Serum samples from healthy controls did not bind to any of the insulin formats (Fig. 5).

Relationship of antibody binding to oxPTM-INS with clinical features Binding of samples from individuals with type 1 diabetes to native insulin and oxPTM-INS was unrelated to indices of metabolic control, such as fasting plasma glucose and $\mathrm{HbA}_{1 c}$, either at diagnosis or after metabolic compensation $(-0.03<\rho<0.16, p>0.132)$, or to fasting C-peptide, insulin requirements or age $(-0.07<\rho<-0.01, p>0.916)$. 
a

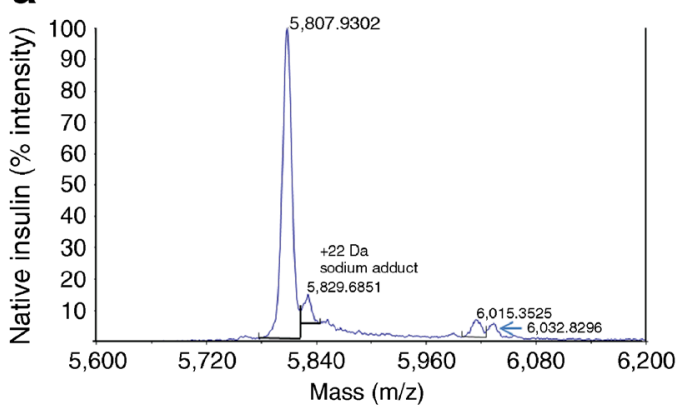

C

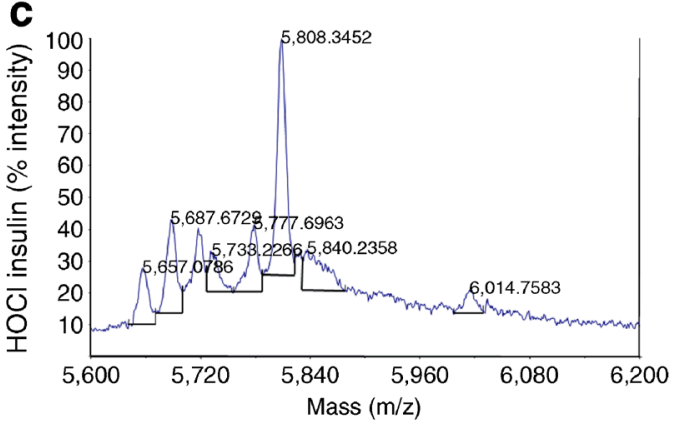

b

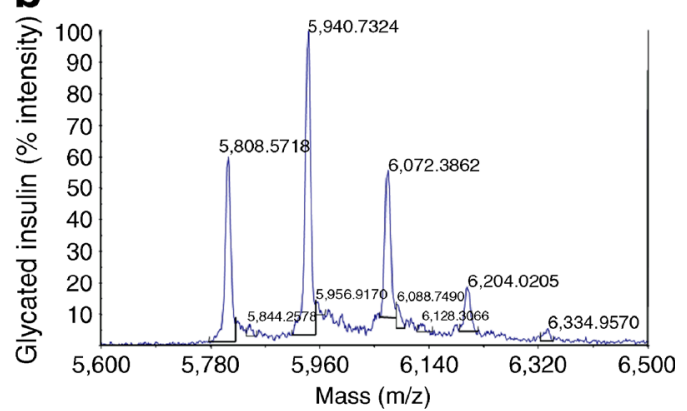

d

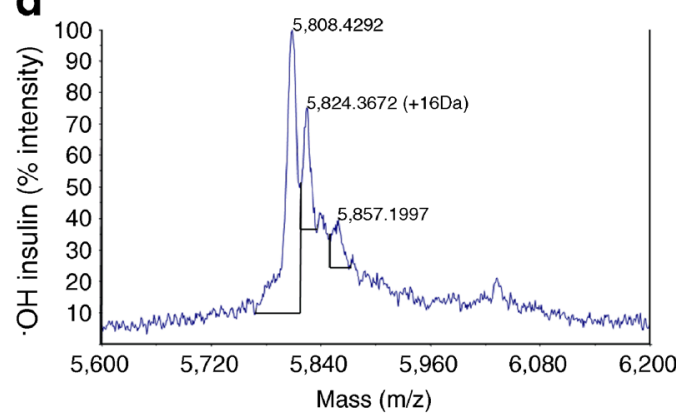

e

Chain A

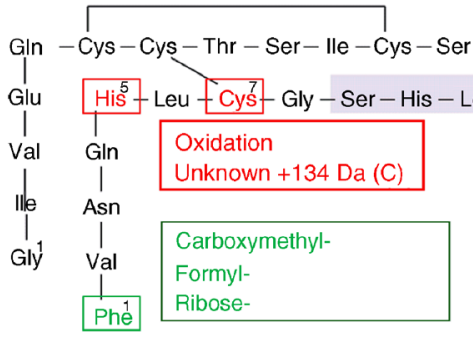

Fig. 2 Analysis of native insulin and oxPTM-INS by MS. (a-d) MALDI-TOF analysis of native insulin and oxPTM-INS. (a) Native insulin showed a peak at m/z 5,808 Da. (b) After glycation by ribose, four additional peaks appeared with mass differences of $132 \mathrm{Da}$ and corresponding to the mono-, di-, tri- and tetra-glycated insulin forms. (c) Exposure to $\mathrm{HOCl}$ induced the development of additional peaks at lower $\mathrm{m} / \mathrm{z}$ than the native form, indicating the formation of degradation products induced by the reaction with the oxidant. (d) A peak at m/z 5,824 Da

\section{Discussion}

This study strengthens the support for the concept of insulin as the prominent autoantigen in type 1 diabetes and suggests that oxPTM neo-antigenic epitopes are involved in insulin reactivity in the large majority of children diagnosed with type 1 diabetes. We have demonstrated, for the first time, that autoreactivity to oxPTM-INS in individuals with newly diagnosed type 1 diabetes is significantly more prevalent than IAAs measured by the gold-standard RBA. Altogether, oxPTM-INS antibodies and IAAs by RBA were detected in $95 \%$ of patients with newly diagnosed type 1 diabetes.

Our data indicate that insulin chain B is the main site of oxPTM. MS mapped oxPTM to chlorination of Tyr16 and Tyr26; oxidation of His5, Cys7 and Phe24; and glycation of
Lys29 and Phe1 in chain B. Our observations correlate with previous studies showing that glycation of insulin involves $\mathrm{N}$ terminal Phe1 and Lys29 [19, 20] in chain B. Similarly, the oxidation in a more central insulin sequence correlates with a previous report implicating Tyr16 and Tyr26, Phe24 and Cys19 as the preferred sites of changes [20]. It is interesting to note that one of the observed modifications involving chlorination of Tyr16 of chain B (Tyr B16) is within the region 9-23 of chain B (B:9-23), a known dominant epitope in autoimmune diabetes [21], with Tyr16 being crucial in immunoreactivity. Substitution of Tyr in position 16 to Ala (Tyr B16:Ala) has been shown to abrogate T cell reactivity [22,23], and administration of a modified B:9-23 (Tyr B16:Ala) peptide has been reported to suppress expression of IAA and prevent diabetes in the NOD mouse [24]; conversely, it is possible that Tyr16 modification by $\mathrm{HOCl}$ 
Table 2 MS/MS analysis of insulin chain B. The table shows the main oxPTM involving the peptides B:1-13

(FVNQHLCGSHLVE), B:14-21

(ALYLVCGE) and B:21-30 (ERGFFYTPKT). No changes were detected in chain A

\begin{tabular}{|c|c|}
\hline $\mathrm{MH}+$ found & Sequence \\
\hline \multicolumn{2}{|l|}{ Native insulin } \\
\hline 1482.7268 & -.FVNQHLCGSHLVE.A \\
\hline 1514.6863 & -.FVNQHLCGSHLVE.A+dioxidation (C) \\
\hline 867.43 & ALYLVCGE \\
\hline 1116.5885 & E.RGFFYTPKT.G \\
\hline \multicolumn{2}{|l|}{ Glycated insulin } \\
\hline 1482.6830 & -.FVNQHLCGSHLVE.A \\
\hline 1510.6870 & -.FVNQHLCGSHLVE.A+formyl (N-terminal) \\
\hline 1540.68 & -.FVNQHLCGSHLVE.A+carboxymethyl (N-terminal) \\
\hline 1614.73 & -.FVNQHLCGSHLVE.A+D-ribose (N-terminal) \\
\hline 1116.5745 & E.RGFFYTPKT.G \\
\hline 1144.5599 & E.RGFFYTPKT.R+formyl (K) \\
\hline 1174.5616 & E.RGFFYTPKT.R+carboxymethyl (K) \\
\hline 1248.61 & E.RGFFYTPKT.R+D-ribose \\
\hline 1194.5848 & E.RGFFYTPKT.R+D-ribose $-3 \mathrm{H}_{2} \mathrm{O}$ \\
\hline \multicolumn{2}{|l|}{${ }^{\circ} \mathrm{OH}-\mathrm{INS}$} \\
\hline 1482.6886 & -.FVNQHLCGSHLVE.A \\
\hline 1498.6687 & -.FVNQHLCGSHLVE.A+oxidation (HW) \\
\hline 1514.6658 & -.FVNQHLCGSHLVE.A+oxidation (C); oxidation (HW) \\
\hline 1514.6863 & -.FVNQHLCGSHLVE.A+dioxidation (C) \\
\hline 1530.68 & -.FVNQHLCGSHLVE.A+trioxidation (C) \\
\hline 1116.5701 & E.RGFFYTPKT.G \\
\hline 1132.5 & RGFFYTPKT+oxidation (F) \\
\hline \multicolumn{2}{|l|}{ HOCl-INS } \\
\hline 1482.7009 & -.FVNQHLCGSHLVE.A \\
\hline 1530.6876 & $\begin{array}{l}\text {-.FVNQHLCGSHLVE.A+oxidation (C); oxidation (F); } \\
\text { oxidation (HW) }\end{array}$ \\
\hline 1530.6876 & -.FVNQHLCGSHLVE.A+trioxidation (C) \\
\hline 867.43 & ALYLVCGE \\
\hline 901.39 & ALYLVCGE +3-chlorotyrosine \\
\hline 1116.5859 & E.RGFFYTPKT.G \\
\hline 1150.5411 & RGFFYTPKT +3-chlorotyrosine \\
\hline 1184.4957 & RGFFYTPKT +3,5-dichlorotyrosine \\
\hline
\end{tabular}

might enhance immunogenicity of this insulin epitope. It is also possible that the other oxidative changes induced by ${ }^{\circ} \mathrm{OH}$ and $\mathrm{HOCl}$ generate neoepitopes as a result of structural changes or by exposing native hidden amino acid sequences.

oxPTM by ROS appears to play a key role in the pathogenesis of several human autoimmune diseases [13, 25-27]. This is of particular relevance to type 1 diabetes, where islet inflammation and the ensuing hyperglycaemia are substantial sources of ROS production [2, 5]. In this regard, beta cells are very susceptible to oxidative damage. Levels of oxygen radical scavenger enzymes are physiologically lower in beta cells compared with other cell types [28], and exposure to cytokines during insulitis might further disrupt the intracellular redox state by enhancing ROS production. The high prevalence of reactivity to oxPTM-INS in the present study indicates that the imbalance of the redox state takes place in a large proportion of patients with type 1 diabetes, and is therefore potentially relevant to disease pathogenesis. Hyperglycaemia can unbalance the redox status later in the disease and contribute to a vicious cycle by providing additional neoepitopes to which the immune system is not tolerant. Indeed, insulin glycation has been detected both in the pancreas in vivo [29] and in the serum of individuals with diabetes [30]. Interestingly, we did not observe increased reactivity to glycated insulin in our study. This is in contrast to the increased autoreactivity to glycated collagen type II that we have previously observed [7, 13]. Although this might imply that imbalance of the redox state and hence ROS production is more 
Fig. 3 Binding of type 1 diabetes serum to native insulin (NT-INS) and oxPTM-INS. (a) ELISA binding to insulin by serum samples from patients with type 1 diabetes, patients with type 2 diabetes and healthy controls. Reactivity to NT-INS and oxPTM-INS was significantly higher in samples from those with type 1 diabetes compared with type 2 diabetes and healthy controls $(p<0.001)$. Binding to oxPTM-INS was significantly higher than to NT-INS in samples from participants with type 1 diabetes $(p<0.0001)$. (b) Levels of IAA were assessed by RBA in the same group of patients with type 1 diabetes and healthy schoolchildren. (c-e)

Longitudinal changes in antibody binding to (c) NT-INS $(p<0.0001)$ and (d) HOCl-INS $(p=0.007)$ by ELISA and (e) insulin antibodies assessed by RBA $(p<0.0001)$, before and after insulin therapy a

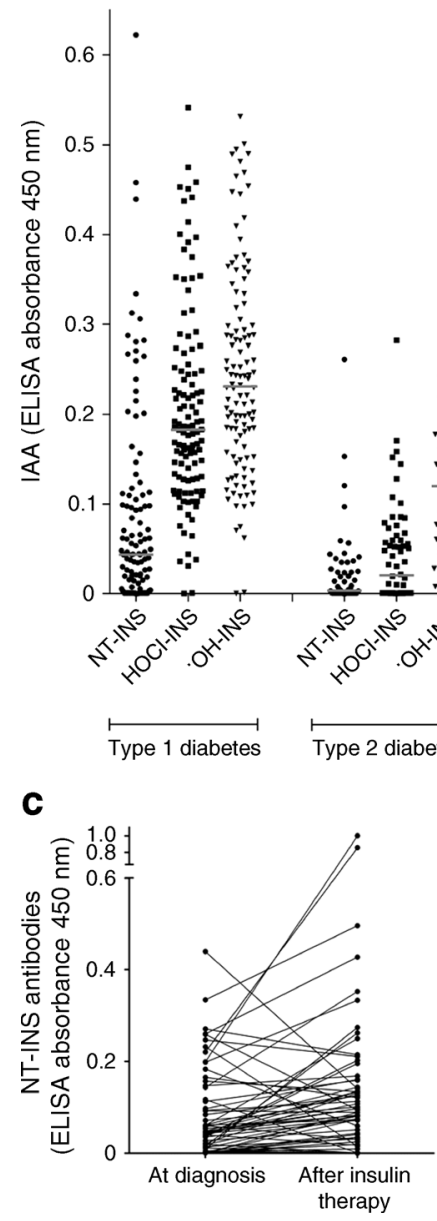

b

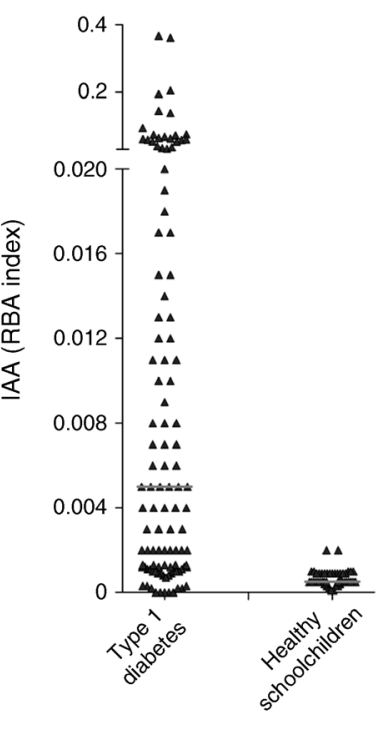

likely inducing the formation of insulin neoepitopes, the interplay between hyperglycaemia (and the substantial contribution
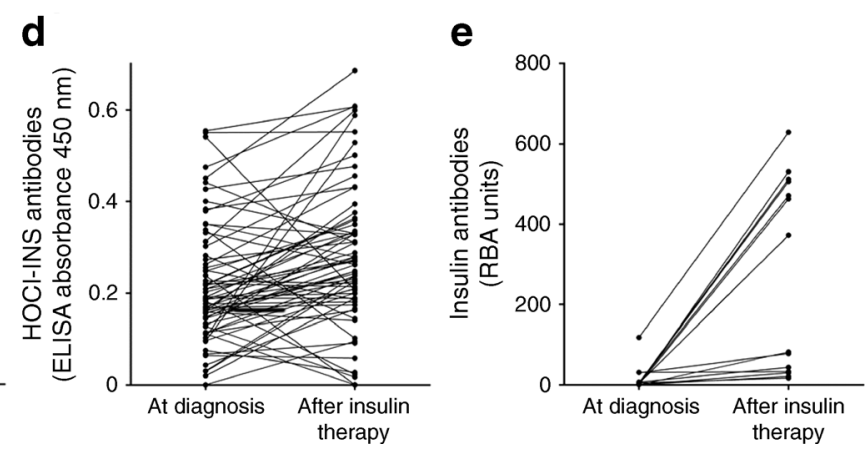

of glycoxidation to the formation of ${ }^{\circ} \mathrm{OH}$ ) and $\mathrm{ROS}$ in inducing oxPTM-INS should be investigated in a separate study.
Fig. 4 Serum binding specificity to oxPTM-INS. Pre-incubation of type 1 diabetes serum samples with oxPTM-INS, but not with native insulin (NT-INS), strongly inhibited binding to oxPTM-INS, indicating the presence of antigen-binding sites specific to oxPTM-INS. Data are shown for insulin modified by ${ }^{\circ} \mathrm{OH}$, as an example of oxPTM-INS a
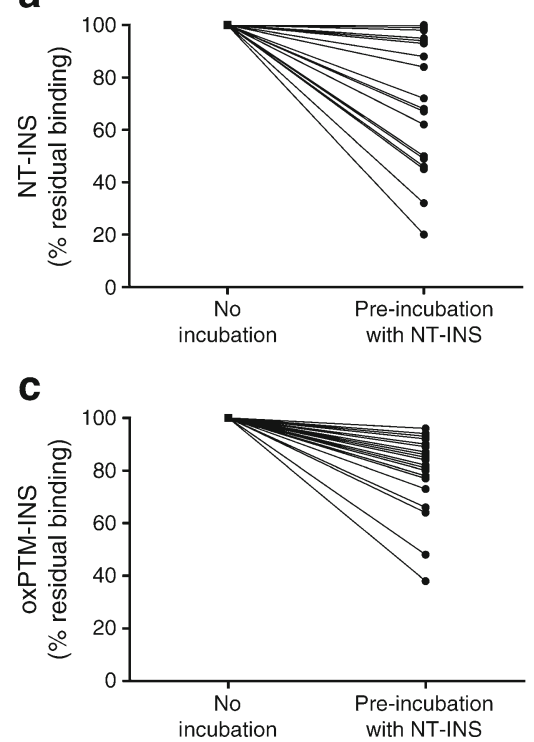

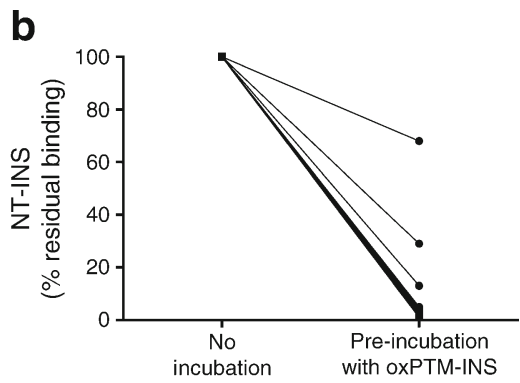

d

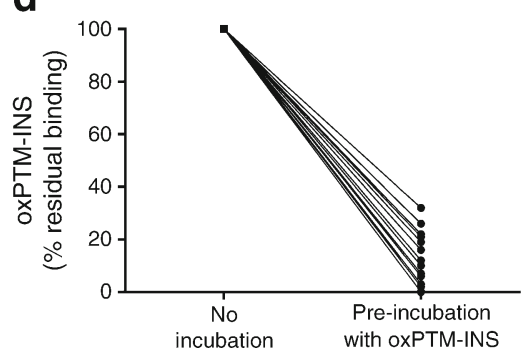




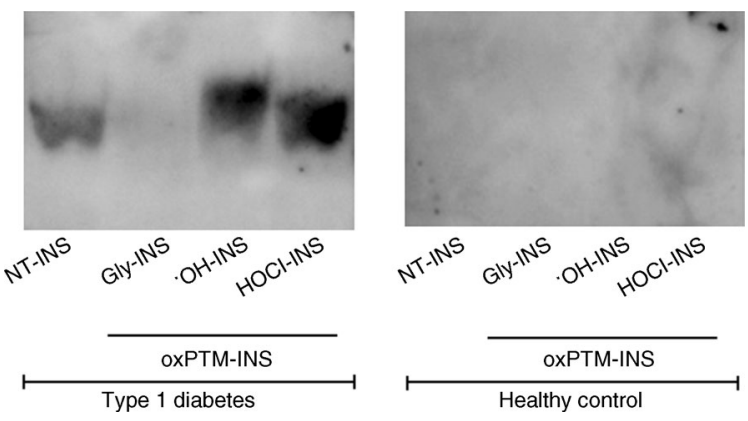

Fig. 5 Binding to native insulin (NT-INS) and oxPTM-INS, as detected by western blot. Binding to NT-INS and a stronger-intensity binding to a smear of a smaller mobility fragment of ${ }^{\circ} \mathrm{OH}-\mathrm{INS}$ and HOCl-INS was observed. No binding to Gly-INS was seen

In this study, we developed an ELISA for detecting autoreactivity to oxPTM-INS. Historically, ELISAs have shown lower performance than liquid-phase assays such as RBA in detecting IAAs [31]. Discrepancies between the techniques have been related to differences in binding affinity and conformational changes induced by the binding of insulin to the plastic in ELISA [32]. However, while being the reference method for IAAs [31], development of an RBA method for oxPTM-INS might not be straightforward, as fragments resulting from oxPTM might lack the amino acids required for radiolabelling, and a complex labelling approach might need to be developed. Furthermore, reactivity in RBA might not be straightforward, as insulin sites for radiolabelling are also potential sites for oxidation. Future studies should address the feasibility of RBA for detecting oxPTM-INS antibodies.

In summary, the present study provides proof of concept for a role of oxPTM in islet autoimmunity, and suggests antioxPTM-INS autoreactivity as a novel immune response in type 1 diabetes. Additional studies with larger cohorts (cross-sectional, longitudinal and at-risk individuals) are required to further study the role of oxPTM in the pathogenesis of type 1 diabetes and to confirm the potential of anti-oxPTMINS as a diagnostic biomarker in humans with type 1 diabetes. It would be of interest to evaluate whether oxPTM-INS antibodies are also present in patients diagnosed as adults, where IAA are usually rarely detected, and in patients who are classified as autoantibody negative to the other existing markers (GADA, IA-2A, ZnT8A). If proven relevant to the disease pathogenesis, oxPTM might mark diseased-tissue pathways, providing previously unknown targets for the development of drugs, biomarkers and imaging techniques.

Acknowledgements We thank N. Nayuni (BioAnalytical Science, Queen Mary University of London, London, UK) for his help with the 3D fluorescence profile, and E. Bland (Innovation, Queen Mary University of London) and D. Cooper (Biochemical Pharmacology, Queen Mary University of London) for reviewing the manuscript.
Funding RS was in part supported by the EFSD Albert Renold Fellowship and the Fondazione Livio Patrizi SIMI award. CV was supported by the $\mathrm{PhD}$ programme of University Campus Bio-Medico and Queen Mary University. This study was in part supported by research grants to NN (Ministero della Salute, Giovani Ricercatori 20091607545) and PP (Ministero Universitá e Ricerca Scientifica, Progetti di Interesse Nazionale PRIN 2010).

Duality of interest The authors declare that there is no duality of interest associated with this manuscript.

Contribution statement RS was responsible for the conception and design of the study, data acquisition, analysis and interpretation of data, and writing the manuscript and revising it critically for important intellectual content. CV and MHA were responsible for data acquisition and revised the manuscript critically for important intellectual content. DP, $\mathrm{CT}, \mathrm{FC}$ and $\mathrm{NN}$ made substantial contributions to data acquisition and revised the manuscript critically for important intellectual content. PP made a substantial contribution to the conception and design of the study, and critically revised the manuscript for important intellectual content. AN made substantial contributions to the conception and design of the study, analysis and interpretation of data, and writing the manuscript, and revised the manuscript critically for important intellectual content. All authors approved the final version. RS and AN are the guarantors of this work and, as such, had full access to all the data in the study and take responsibility for the integrity of the data and the accuracy of the data analysis.

\section{References}

1. Eggleton P, Nissim A, Ryan BJ, Whiteman M, Winyard PG (2013) Detection and isolation of human serum autoantibodies that recognize oxidatively modified autoantigens. Free Radic Biol Med 57:79-91

2. West IC (2000) Radicals and oxidative stress in diabetes. Diabet Med 17:171-180

3. Ryan BJ, Nissim A, Winyard PG (2014) Oxidative posttranslational modifications and their involvement in the pathogenesis of autoimmune diseases. Redox Biol 2:715-724

4. Storling J, Overgaard AJ, Brorsson CA et al (2013) Do posttranslational beta cell protein modifications trigger type 1 diabetes? Diabetologia 56:2347-2354

5. Eizirik DL, Cnop M (2010) ER stress in pancreatic beta cells: the thin red line between adaptation and failure. Sci Signal 3:pe7

6. Sollid LM, Jabri B (2011) Celiac disease and transglutaminase 2: a model for posttranslational modification of antigens and HLA association in the pathogenesis of autoimmune disorders. Curr Opin Immunol 23:732-738

7. Strollo R, Ponchel F, Malmstrom V et al (2013) Autoantibodies to posttranslationally modified type II collagen as potential biomarkers for rheumatoid arthritis. Arthritis Rheum 65:1702-1712

8. van Lummel M, Duinkerken G, van Veelen PA et al (2014) Posttranslational modification of HLA-DQ binding islet autoantigens in type 1 diabetes. Diabetes 63:237-247

9. McGinty JW, Chow IT, Greenbaum C, Odegard J, Kwok WW, James EA (2014) Recognition of post-translationally modified glutamic acid decarboxylase 65 epitopes in subjects with type 1 diabetes. Diabetes 63:3033-3040

10. Mannering SI, Harrison LC, Williamson NA et al (2005) The insulin A-chain epitope recognized by human $\mathrm{T}$ cells is posttranslationally modified. J Exp Med 202:1191-1197

11. Trigwell SM, Radford PM, Page SR et al (2001) Islet glutamic acid decarboxylase modified by reactive oxygen species is recognized 
by antibodies from patients with type 1 diabetes mellitus. Clin Exp Immunol 126:242-249

12. Rondas D, Crevecoeur I, D'Hertog W et al (2015) Citrullinated glucose-regulated protein 78 is an autoantigen in type 1 diabetes. Diabetes 64:573-586

13. Strollo R, Rizzo P, Spoletini M et al (2013) HLA-dependent autoantibodies against post-translationally modified collagen type II in type 1 diabetes mellitus. Diabetologia 56:563-572

14. Palmer JP, Asplin CM, Clemons P et al (1983) Insulin antibodies in insulin-dependent diabetics before insulin treatment. Science 222:1337-1339

15. Zhang L, Eisenbarth GS (2011) Prediction and prevention of type 1 diabetes mellitus. J Diabetes 3:48-57

16. Karjalainen J, Salmela P, Ilonen J, Surcel HM, Knip M (1989) A comparison of childhood and adult type I diabetes mellitus. N Engl J Med 320:881-886

17. Al Alwan I, Bin Dajim N, Jawdat D, Tamimi W, Al Ahmdi R, Albuhairan F (2012) Prevalence of autoantibodies in children newly diagnosed with type 1 diabetes mellitus. Br J Biomed Sci 69:31-33

18. Williams AJ, Bingley PJ, Bonifacio E, Palmer JP, Gale EA (1997) A novel micro-assay for insulin autoantibodies. J Autoimmun 10:473-478

19. Hunter SJ, Boyd AC, O'Harte FP et al (2003) Demonstration of glycated insulin in human diabetic plasma and decreased biological activity assessed by euglycemic-hyperinsulinemic clamp technique in humans. Diabetes 52:492-498

20. Guedes S, Vitorino R, Domingues MR, Amado F, Domingues P (2010) Oxidative modifications in glycated insulin. Anal Bioanal Chem 397:1985-1995

21. Nakayama M, Abiru N, Moriyama $\mathrm{H}$ et al (2005) Prime role for an insulin epitope in the development of type 1 diabetes in NOD mice. Nature 435:220-223

22. Abiru N, Wegmann D, Kawasaki E, Gottlieb P, Simone E, Eisenbarth GS (2000) Dual overlapping peptides recognized by insulin peptide B:9-23 T cell receptor AV13S3 T cell clones of the NOD mouse. J Autoimmun 14:231-237
23. Wong FS, Moustakas AK, Wen L, Papadopoulos GK, Janeway CA Jr (2002) Analysis of structure and function relationships of an autoantigenic peptide of insulin bound to $\mathrm{H}-2 \mathrm{~K}(\mathrm{~d})$ that stimulates CD8 T cells in insulin-dependent diabetes mellitus. Proc Natl Acad Sci U S A 99:5551-5556

24. Kobayashi M, Abiru N, Arakawa T et al (2007) Altered B:9-23 insulin, when administered intranasally with cholera toxin adjuvant, suppresses the expression of insulin autoantibodies and prevents diabetes. J Immunol 179:2082-2088

25. Nissim A, Winyard PG, Corrigall V et al (2005) Generation of neoantigenic epitopes after posttranslational modification of type II collagen by factors present within the inflamed joint. Arthritis Rheum 52:3829-3838

26. Hendler A, Mulli TK, Hughes FJ et al (2010) Involvement of autoimmunity in the pathogenesis of aggressive periodontitis. J Dent Res 89:1389-1394

27. Otaki N, Chikazawa M, Nagae R et al (2010) Identification of a lipid peroxidation product as the source of oxidation-specific epitopes recognized by anti-DNA autoantibodies. J Biol Chem 285:33834-33842

28. Acharya JD, Ghaskadbi SS (2010) Islets and their antioxidant defense. Islets 2:225-235

29. Abdel-Wahab YH, O'Harte FP, Ratcliff H, McClenaghan NH, Barnett CR, Flatt PR (1996) Glycation of insulin in the islets of Langerhans of normal and diabetic animals. Diabetes 45:1489-1496

30. Lindsay JR, McKillop AM, Mooney MH, O'Harte FP, Bell PM, Flatt PR (2003) Demonstration of increased concentrations of circulating glycated insulin in human type 2 diabetes using a novel and specific radioimmunoassay. Diabetologia 46:475-478

31. Schlosser M, Mueller PW, Torn C, Bonifacio E, Bingley PJ (2010) Diabetes Antibody Standardization Program: evaluation of assays for insulin autoantibodies. Diabetologia 53:2611-2620

32. Brezar V, Carel JC, Boitard C, Mallone R (2011) Beyond the hormone: insulin as an autoimmune target in type 1 diabetes. Endocr Rev 32:623-669 